
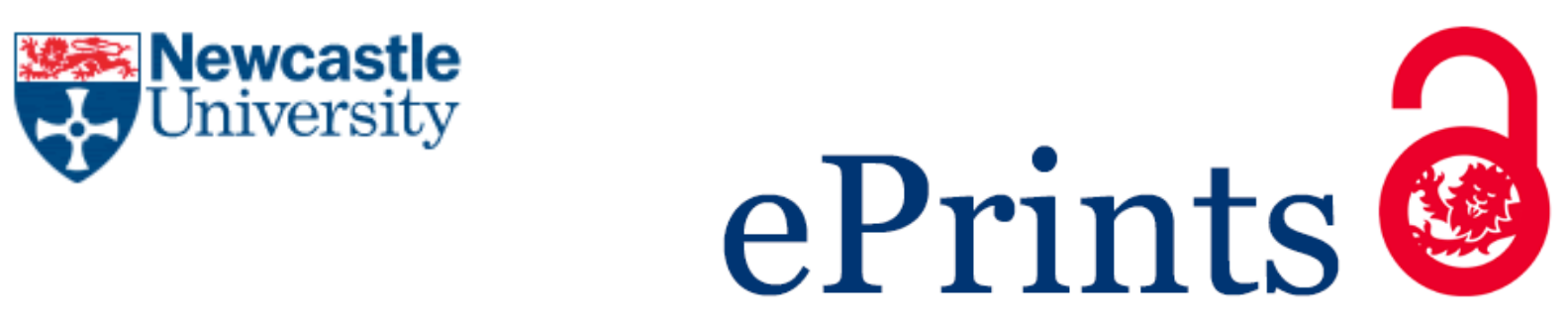

Abdullah MAM, Dlay SS, Woo WL. Fast and Accurate Pupil Isolation Based on Morphology and Active Contour. In: 4th International conference on Signal, Image Processing and Applications. 2014, Nottingham, UK. Published in: International Journal of Information and Electronics Engineering, 2010-3719, Volume 4, Number 6, 2014 by IACSIT.

Copyright:

(C) International Journal of Information and Electronics Engineering 2014.

DOI link to article:

http://dx.doi.org/10.7763/IJIEE.2014.V4.476

Date deposited:

$06 / 07 / 2015$

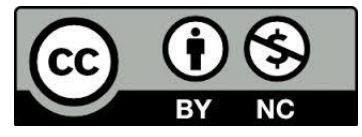

This work is licensed under a Creative Commons Attribution-NonCommercial 3.0 Unported License 


\title{
Fast and Accurate Pupil Isolation Based on Morphology and Active Contour
}

\author{
Mohammed A. M. Abdullah, S. S. Dlay, and W. L. Woo
}

\begin{abstract}
The performance of iris recognition systems is significantly affected by the segmentation accuracy, especially in non-ideal iris images. This paper proposes a fast and accurate method to segment the pupil regardless of the shape using a combination of morphological operations and snake active contour. The morphological operations are used to determine a rough boundary of the pupil region while the active contour is used to find the precise boundary. The proposed scheme is robust in finding the exact pupil boundary of non-ideal iris image. Experimental results on the CASIA version 4.0 iris databases have indicated a high level of accuracy using the proposed technique.
\end{abstract}

Index Terms - active contour, iris recognition, image segmentation, morphological operations, pupil segmentation.

\section{INTRODUCTION}

Biometrics are automated methods of recognizing a person based on one or multiple physical or behavioral characteristics. Among these biologic characteristics, iris pattern has gained an increasing amount of attention because it is one of the most accurate and reliable human identification techniques. Also, iris patterns possess a high degree of randomness and uniqueness even between identical twins and remain constantly stable throughout adult's life $[1,2]$. A common iris image is depicted in Fig. 1.

Generally, an iris recognition system comprises of four main stages: iris segmentation, iris normalization, feature extraction and feature matching [3]. Iris segmentation is the first and probably the most important step in an iris recognition system as the performance of the system is highly dependent on this step and errors can lead to misclassification during authentication. This step involves isolating an iris from other components in an eye image. Generally, an inner boundary of the iris is defined by a boundary of the pupil while the outer boundary of the iris is a boundary between the white sclera and the iris.

Many applications require accurate pupil features detection such as iris recognition, gaze detection and disease diagnosis. For instance, Proenca and Alexandre [4] observed a significant degradation of iris recognition rates, especially in the presence of translation errors of the segmented pupil border.

Manuscript received March 19, 2014; revised March 31, 2014.

The authors are with the school of Electrical and Electronic Engineering, Newcastle University, England, United Kingdom.

Mohammed A. M. Abdullah is also a staff member with the University of Mosul in Nineveh, Iraq (email: mabdul@uomosul.edu.iq).

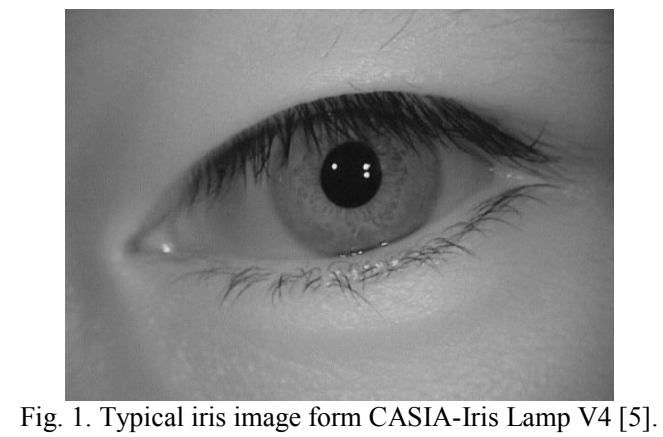

According to Huang et al. [6], the actual pupil boundary are not circular, while the outer iris boundary is close to a circle. In addition, iris rich textures are closer to the pupil than to the sclera [7]. These two facts explain the importance to accurately segment the inner iris boundary.

In this paper, we propose a robust pupil segmentation algorithm based on an active contour model and morphological operations. Experimental results show that our method has superior performance in term of accuracy and complexity.

The remainder of this paper is organized as follow: in the next section, we present a literature review on iris segmentation; following this we introduce the proposed method and its implementation. Then, the results are presented and discussed, finally the conclusions are presented.

\section{LITERATURE REVIEW}

Numerous iris segmentation methods have been proposed in literature. Amongst, there are two well-known algorithms proposed by Daugman [8] and Wildes [9]. Daugman, in [8], applied an integro-differential operator to delimitate the circular boundaries of the iris, while Wildes in [9] used Hough transforms to locate iris boundaries. Both algorithms give good performances but have a major disadvantage in term of computational cost as they need to search the whole image.

Other methods based on circular iris boundaries approximation also have been implemented such as least square method proposed by Zhu et al. [10] and gray-scale distribution feature method proposed by Yuan et al. [11]. However, all these methods above locate the pupil considering it as a standard circle which causes errors in term of location accuracy.

Ryan et al. [12] utilized the Starburst algorithm to detect limbic and pupil boundary. Good results in iris segmentation are reported but the number of parameters to adjust and search increased (major and minor axis length, center coordinates and rotation angle). 
In [13] intensity information is used to find a square region that completely surrounds the pupil. The square region is then binarized to extract an edge map. Iterative morphological operations are then applied to detect the iris inner boundary. Obtained results show an improvement in the precision of the iris localization. But it must also be noted that the database used to test the methodology is CASIA V1 which considered as a non-challenging database.

Recently, several researchers proposed different unideal iris recognition schemes. For example, Talebi, S. M. et al. [14] used a balloon active contour to segment the iris image form CASIA V1. However, the drawback with this method is that the initial contour is set manually.

On the other hand, others [15-17] proposed an automated method to estimate the rough boundaries of the iris then implement the active contour to find the accurate pupil and iris boundaries. As a case in point, [15] applied direct least square based elliptical fitting to obtain an initial approximation of the pupil and the iris boundaries. Similarly, $[16,17]$ take advantage of the circular Hough transform to initialize the active contour. Although these methods achieved good results, the main limitation is the complexity of the method used to find the initial curve comparing it to the simple morphological operations that we used in this paper.

\section{The SNAKe Active CONTOUR Model}

The active contour model, also called snake, which is based on the energy function was introduced by Kass et al. [18]. The snake has become popular in computer vision fields such as edge extraction, image segmentation, motion tracking and 3D reconstruction. A snake or an active contour is a parametric curve which evolves from an initial state into a position where its energy is minimized under the influences of internal forces, image forces and external constraint forces.

The snake is represented by $v(s)=[x(s), y(s)]$ where $s$ represents the length of the curve. The snake energy is defined as:

$$
E_{\text {snake }}=\int_{0}^{1} E_{\text {int }}(v(s)) d s+\int_{0}^{1} E_{\text {ext }}(v(s)) d s
$$

where $E_{\text {int }}(v(s))$ represents the internal energy and $E_{\text {ext }}(v(s))$ represents the external energy of the snake. The internal energy is used to control the deformability of the snake, and is written as:

$$
E_{\text {int }}(v(s))=\frac{1}{2}\left(\alpha(s)\left|v_{s}(s)\right|^{2}+\beta(s)\left|v_{s s}(s)\right|^{2}\right)
$$

Here $\boldsymbol{V}_{\boldsymbol{s}}$ is the first spatial derivative and $\boldsymbol{V}_{\boldsymbol{s}}$ is the second spatial derivative of the snake's parameters (coordinates). Therefore, the first term represents elasticity, or membrane-like behavior, while the second term represents rigidity or thin-plate behavior. The coefficients, $\alpha$ and $\beta$, can be used to control the continuity characteristics of the snake by changing its elasticity and rigidity. For example, setting $\beta(s)$ to zero at a point allows the snake to become second-order discontinuous and develop a corner.

The external energy $E_{\text {ext }}(v(s))$ is composed of image energy.

$$
E_{\text {ext }}(v(s))=\gamma(s) E_{\text {image }}(v(s))
$$

where $\gamma$ controls the energy term. The image energy is used to represent the structure data of the image such as intensity and gradient value. For example, in the case where edges of the image are of interest, the image energy can be defined as:

$$
E_{\text {image }}(x, y)=-\left|\nabla\left(G_{\sigma}(x, y) * I(x, y)\right)\right|^{2}
$$

where $G_{\sigma}(x, y)$ is a Gaussian function and * represents the convolution while $I(x, y)$ is the image intensity function. This convolution smoothes the image by a Gaussian function to eliminate noise.

Substituting the $\operatorname{Eint}(\mathrm{v}(\mathrm{s}))$ into the energy function, we finally get the whole expression of the snake:

$$
E_{\text {snake }}=\int_{s}\left(\alpha(s)\left|v_{s}(s)\right|^{2}+\beta(s)\left|v_{s s}(s)\right|^{2}\right)+\gamma(s) E_{\text {image }}(v(s)) d s
$$

and with the integrals having the form $F\left(v(s), v^{\prime}(s), v^{\prime \prime}(s)\right)$, a necessary condition for a function to minimize (5) is that it satisfies the following Euler equation:

$$
\alpha(s) v_{s s}+\beta(s) v_{s s s s}-\nabla E_{\text {image }}=0
$$

Solution of the above equation gives the final contour minimizing $E_{\text {snake }}$. where $\nabla$ is the gradient operator and this equilibrium function could be considered as the force equilibrium function is written as:

$$
F_{\text {int }}+F_{\text {ext }}=0
$$

The internal forces discourage stretching and bending while the external force pulls the snake towards the desired image edges. Therefore, when the original contour evolves and deforms into the final contour $F_{\text {int }}=-F_{\text {ext }}$ which means that for every point along the curve the internal and external forces are equal and act in opposite direction to each other giving a stable state. Details of the minimization procedure are given in [18].

\section{Proposed Methodology}

The proposed method of pupil detection in this paper is designed to localize pupillary boundary accurately. More specifically, the module is broadly consisting of the following steps, namely: $(A)$ reflection removal, $(B)$ binary image generation by thresholding, $(C)$ eyelashes removal, $(D)$ rough pupil's boundary estimation and $(E)$ accurate pupil segmentation with active contour.

A detailed description of the signal processing procedures involved in each step is given below. For the illustration purposes, Fig. 2 shows the major involved processing procedures and the obtained results. In this section the proposed method will be discussed in details.

\section{A. Reflection Removal}

As in the iris image shown in Fig. 1 the pupil region has some light reflection spots caused by the near infrared illuminators used to collect the iris images of CASIA-Iris Lamp V4 database. Since these reflection spots cause false pupil boundary detection, a technique is required to remove these artifacts.

To address this problem, a simple procedure is adopted; the spots of reflections are removed by thresholding the eye image with a threshold value of 240 . This is because the light reflections usually have very high intensity values. 


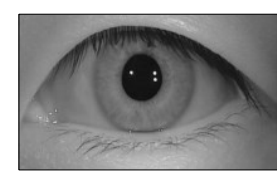

(a)

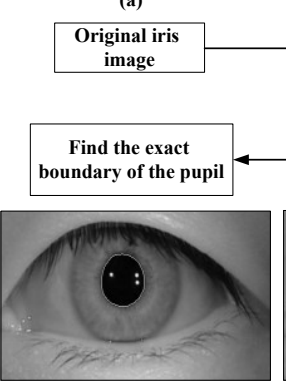

(f)

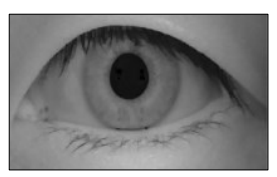

(b)

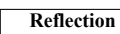

Reflection
removal

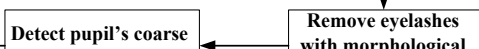
with morphological center and boundary

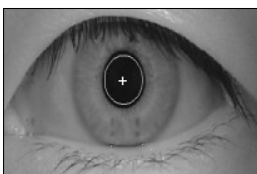

(e)

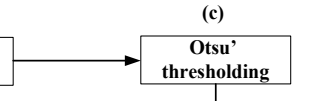

,
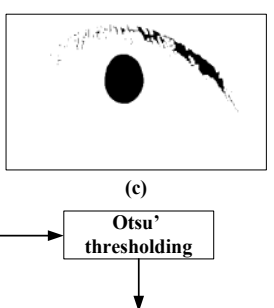

operations
Fig. 2. Flow chart of the pupillary detection algorithm. The major processing procedures, namely, (a) Original image, (b) Reflection removal, (c) Image binarization, (d) Eyelashes removal, (e) Pupil's coarse center and boundary estimation and (f) Pupil isolation with active contour.

To remove the remaining reflection points, the image opening is performed over the pupil region. All edge pixels inside these detected light spot regions are filled. As shown in Fig. 2 (b), the reflections in the image have been eliminated thereby improving the quality of the binary image for later stages.

\section{B. Thresholding}

Generally, iris images are captured under different illumination settings. Selecting a good threshold value by user is possible only after several experimentations. Furthermore, different images from the same iris database can be captured under different illumination settings. Therefore, it is necessary to find an automatic thresholding method to overcome such difficulties.

In this paper, Otsu's method [19] is used for thresholding. Hence, instead of using a given threshold value, the process of extracting it from the image can be automated. Experiments on CASIA-Iris Lamp V4 showed that quarter of the Otsu threshold value, perfectly fits for this purpose. The method supposes that the image to be thresholded contains two types of points such as foreground and background. Computation of the best threshold divides those two regions so their combined spread is minimal.

Fig. 3(a) shows sample an eye image before any processing. Fig. 3(b,c) depicts iris images thresholded with Otsu and quarter of Otsu value respectively. The process of selecting Otsu threshold value is given in Fig. 3(d). Here the right vertical line demonstrates Otsu threshold value, and the left vertical dotted line demonstrates quarter of Otsu threshold value. In some cases of thresholding, Otsu value by itself separates sclera from the rest of the eye in the image. So, taking quarter of the Otsu value is a good approximation for separating pupil from the sclera region.

\section{Eyelashes Removal}

Eye image is very likely to have cases where the lashes are as dark as the pupil, when they appear in the resulting binary image, their presence hinders pupil isolation. To cure this problem, the image is refined in several steps using morphological operations in order to obtain a reliable binary image of the pupil. Firstly, a morphological dilation operation with $8 \times 8$ square structuring element is applied to the binary image in order to eliminate the effect of eyelashes. (a)
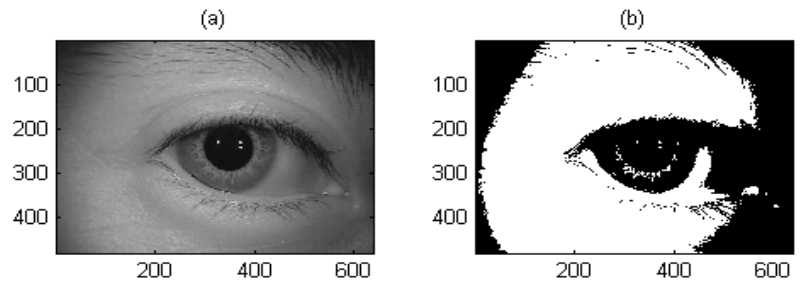

(c)
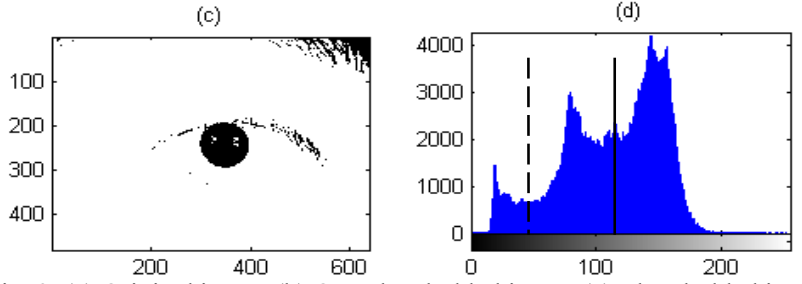

Fig. 3. (a) Original image (b) Otsu thresholded image (c) Thresholded image with quarter-Otsu value (d) Quarter-Otsu (leftmost line) and Otsu values marked on the histogram of the original image.

Secondly, in order to keep only the pupil in the binary image and remove the remaining eyelashes, we employ, as structuring element, a disc of radius slightly lower than the smallest pupil radius in the iris database. Results of this efficient processing are shown in Fig. 2(d).

\section{Detecting Pupil's Coarse Center and Radius}

Once the pupil is localized, the binary image can be used to detect the pupil's coarse parameters. To calculate the center of the pupil the center of mass is calculated. Let $\left(x_{i} y_{i}\right)$, $i=1 \ldots N$ denote the image domain coordinates of the remaining pixels. The center $\left(x_{c}, y_{c}\right)$ of the pupil is obtained by calculating the center of mass of the pixels:

$$
\left(x_{c}, y_{c}\right)=\frac{1}{N} \sum_{i=1}^{N}\left(x_{i}, y_{i}\right)
$$

where $N$ is the total number of black pixels. Indeed, the pupil can be approximated as a circle to determine its coarse radius. With such an approximation, the pupil's radius is directly obtained by recalling that the number of pixels is equal to the area of the circular pupil. Thus,

$$
r=\sqrt{\frac{N}{\Pi}}
$$

\section{E. Pupil Accurate Segmentation with Active Contour}

The crucial step of the active contour is the initialization of the model and the choice of the initial curve. The choice of an arbitrarily form can cause the non-convergence of this contour towards the zone of interest.

Because the snake active contour initial curve should be set outside the object boundary, hence, a constant value of 5 is added to radius of the previously estimated circle to make sure that the initial curve always lies outside the pupil boundary so the active contour can converge correctly as shown in Fig. 4.

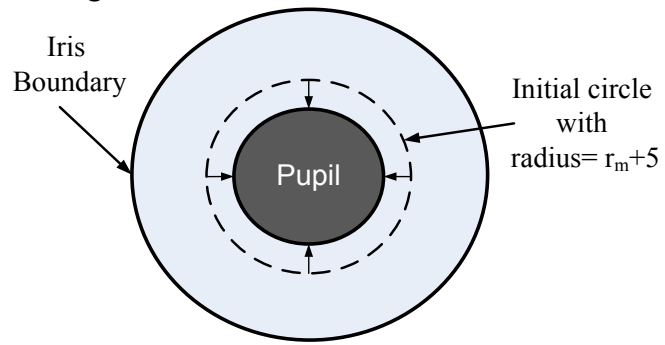

Fig. 4. Initial circle converging toward the boundary of the pupil using active contour, where $r_{m}$ is the radius estimated by morphological operations. 
This, consequently, will improve the accuracy of the final contour and reduce the number of snake iterations. Because of the dark intensity of the pupil region, snake active contour is robust and stable in finding the inner iris boundary.

\section{V.RESULTS AND DISCUSSION}

Experiments conducted on the newest CASIA iris image database (version 4) from the Institute of Automation, Chinese Academy of Sciences [5]. For our experiments, 200 classes with 10 images in each class were randomly chosen form the IrisV4-Lamp subset since they contain challenging iris images with nonlinear deformations and noisy characteristics such as light reflections and eyelash occlusion.

According to (5) to locate the pupil-iris edge, we perform it with a smaller $\alpha$ and a larger $\beta$ since the pupil-iris edge is similar to a circle. Empirical experiments indicate that best value for these parameters are $\alpha=0.2, \beta=0.4$ and $\gamma=0.15$. With such parameters and prior contour initialization, the snake converges easily with maximum of 30 iterations. The snake active contour model with morphological operations yield a $99.5 \%$ inner pupil segmentation results. Error in segmentation occurred when eyelashes or eyelids are covering a large part of the pupil.

Active contour can generate more accurate contour of the pupil boundary than can be achieved by using circular and elliptic approximations. To prove this, the circular Hough transform has been implemented in order to compare it with the active contour performance. As illustrated in Fig. 5, the two segmentation methods are applied on each iris image at the same time. The green circle is the circular Hough transform segmentation while the white contour is result of our proposed segmentation method.

With reference to Fig. 5, it can be clearly seen that the pupil's shape is neither a perfect circle nor a perfect ellipse. Within the green detected circle, a part of the iris region is considered as belonging to the pupil. Such wrong pupil localization influences the recognition performance. On the other hand, the white contour, which is the result of our segmentation, accurately detects the pupil in its real shape. In order to evaluate the efficiency of our method, we compared it with previously reported techniques in terms of accuracy and speed.

As we demonstrated before, the circular approximations such as Integro-differential operator or circular Hough transform is not accurate for pupil segmentation. In term of speed, Integro-differential operators are inherently slower than the approach used in this paper due to the need for fitting circles with varying radii and center coordinates on the image [8].

On the other hand, circular Hough transform is computationally intensive because all the image pixels must be examined in turn and each generates a large number of votes in Hough space [20]. Similarly, using morphological operations to find the initial curve for active contour is more convenient due to their simplicity and speed compared with Hough transform or elliptical fitting proposed in [15-17].

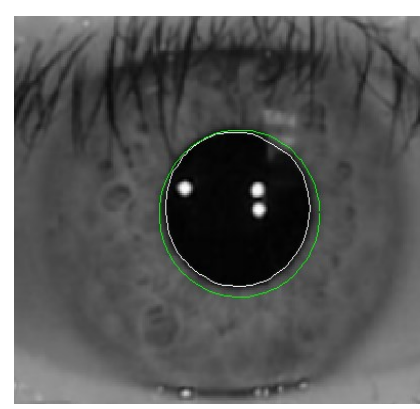

(a) S2005R08

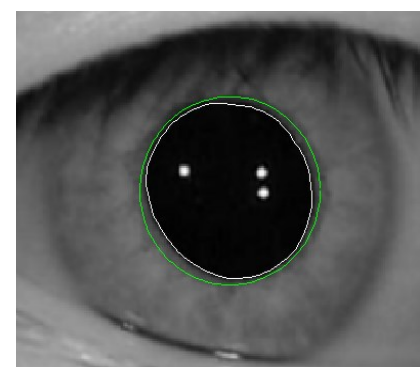

(c) S2012R02

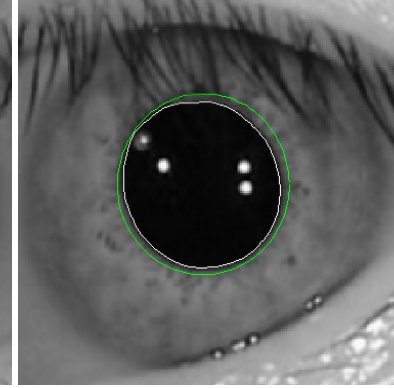

(b) S2007L11

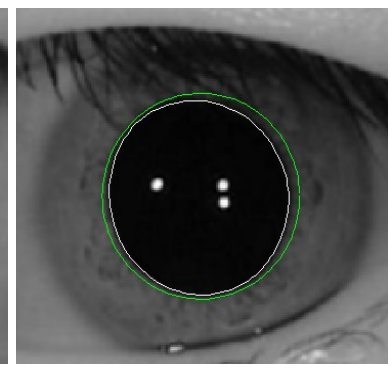

(d) S2052L01
Fig. 5. Performance comparison of circular Hough transform (green circle) and the proposed method (white contour) on four images from CASIA Iris-Lamp V4 namely (a) S2005R08 (b) S2007L11 (c) S2012R02 and (d) S2052L01

Furthermore, for comparison, we applied the well-known methods of $[8,16,17,21]$ for pupil segmentation on the CASIA-Iris Lamp V4. The performance of the pupil segmentation technique was measured by computing the accuracy defined as:

$$
\text { Accuracy }=\frac{\text { No. of correctly segmented pupils }}{\text { Total no. of input pupils }}
$$

As illustrated in Table I, the experimental results verified that our proposed algorithm has a high correct segmentation ratio and lower averaged computational time.

TABLE I: COMPARISON OF DIFFERENT PUPIL SEGMENTATION METHOD
\begin{tabular}{||c|c|c||}
\hline \multicolumn{1}{|c|}{ Algorithm } & Accuracy & $\begin{array}{c}\text { Average Time } \\
\text { (millisecond) }\end{array}$ \\
\hline Proposed Algorithm & $99.5 \%$ & 280 \\
\hline $\begin{array}{l}\text { Integro-differential } \\
\text { operator [8] }\end{array}$ & $90.3 \%$ & 905 \\
\hline $\begin{array}{l}\text { Active contour with } \\
\text { Hough transform [16, 17] }\end{array}$ & $99.0 \%$ & 1503 \\
\hline $\begin{array}{l}\text { Hough transform with } \\
\text { edge detection [21] }\end{array}$ & $95.7 \%$ & 1421 \\
\hline
\end{tabular}

\section{CONCLUSIONS}

In this paper, we proposed an efficient and accurate approach for pupil boundary detection regardless of the pupil's shape. The proposed methodology segments the inner iris boundary in its real shape which will contribute to a more reliable and robust iris recognition system.

For finding the pupil boundary, Otsu's Histogram thresholding and morphological operations have been employed to estimate the rough pupil boundary. Then, a snake active contour is applied to detect the actual boundary. The advantage of the active contour is the generation of a more accurate contour of the pupil boundary than can be achieved using circular and elliptic approximation. 
Given that the pupil is not perfectly circular; such approximations lead to a lose in a part of the texture which is likely to be of a significant discriminating value.

Our tests on a more challenging iris image database set, CASIA-IrisV4-Lamp, confirmed that our segmentation method is robust and results in performance gains. The proposed algorithm has resulted in accurate pupil localization due to precisely locating the center of the pupil and determining its radius. Moreover, the proposed algorithm has been shown to be both robust and efficient as it has successfully isolated the pupil from the iris images. In addition, the algorithm has resulted in performance gains by reducing the computational time by $78.1 \%$ over the average time of the reported algorithms. The proposed method can also be used in medical application for pupil isolation.

Our future works will concentrate on developing algorithms for detecting the outer iris boundary and complete the iris recognition system in order to see the influence on the performance of the iris recognition system.

\section{ACKNOWLEDGMENT}

We are grateful to the Chinese Academy of Sciences, Institute of Automation (CASIA) for providing the iris database.

\section{REFERENCES}

[1] J. Daugman, "High confidence visual recognition of persons by a test of statistical independence," IEEE Transactions on Pattern Analysis and Machine Intelligence, vol. 15, issue 11, pp. 1148-1161, 1993.

[2] M. Li, T. N. Tan, Y. H. Wang, and D. X. Zhang, "Personal identification based on iris texture analysis," IEEE Transactions on Pattern Analysis and Machine Intelligence, vol. 25, issue 12, pp. 1519-1533, 2003.

[3] M. A. M. Abdullah, F. H. A. Al-Dulaimi, W. Al-Nuaimy, and A. Al-Aataby, "Efficient small template Iris recognition system using wavelet transform," International Journal of Biometrics and Bioinformatics (IJBB), vol. 5, pp. 16-27, 2011.

[4] H. Proenca and L. A. Alexandre, "A method for the identification of inaccuracies in pupil segmentation," in Proc. of ARES 2006, 2006.

[5] Chinese Academy of Sciences Institute of Automation. (Accessed on Jan. 2014). CASIA-Iris database V4. Available: http://www.cbsr.ia.ac.cn/china/Iris\%20Databases\%20CH.asp

[6] J. Huang, X. You, Y. Y. Tang, L. Du, and Y. Yuan, "A novel iris segmentation using radial-suppression edge detection," Signal Processing, vol. 89, issue 12, pp. 2630-2643, 2009.

[7] E. Krichen, "Recognition of people by iris in degraded mode," Ph.D, dissertation, Evry-Val Essonne University, France, 2007.

[8] J. Daugman, "Statistical richness of visual phase information: Update on recognizing persons by Iris patterns," International Journal of Computer Vision, vol. 45, pp. 25-38, 2001.

[9] R. P. Wildes, "Iris recognition: an emerging biometric technology," Proceedings of the IEEE, 1997, pp. 1348-1363, vol. 85.

[10] Y. Zhu, T. N. Tan, and Y. H. Wang, "Biometric personal identification based on iris patterns," in Proc. of 15th International Conference on Pattern Recognition, 2000, vol. 2, pp. 801-804.

[11] W. Q. Yuan, L. Xu, Z. H. Lin, "Iris localization algorithm based on gray distribution features of eye images," Journal of Optoelectronics-Laser, vol. 17, 2006.

[12] W. J. Ryan, D. L. Woodard, A. T. Duchowski, and S. T. Birchfield, "Adapting starburst for elliptical Iris segmentation," in Proc. of 2nd IEEE International Conference on Biometrics: Theory, Applications and Systems, 2008, pp. 1-7.

[13] H. Ghodrati, M. J. Dehghani, M. S. Helfroush, and K. Kazemi, "Localization of noncircular iris boundaries using morphology and arched Hough transform," in Proc. of 2010 2nd International Conference on Image Processing Theory Tools and Applications, 2010, pp. 458-463.

[14] S. M. Talebi, A. Ayatollahi, and S. M. S. Moosavi, "A novel Iris segmentation method based on balloon active contour," in Proc. of 2010 6th Iranian Machine Vision and Image Processing, 2010, pp. 1-5.

[15] R. Kaushik, B. Prabir, and C. Y. Sueh, "Unideal iris segmentation using region-based active contour model," in Proc. of ICIAR 2010, 2010, pp. 256-265, Volume Part II.

[16] K. Jaehan, V. Govindaraju, and V. Chaudhary, "A robust Iris localization method using an active contour model and hough transform," in Proc. of 20th International Conference on Pattern Recognition (ICPR), 2010, pp. 2852-2856.

[17] A. Hilal, B. Daya, and P. Beauseroy, "Hough Transform and Active Contour for Enhanced Iris Segmentation," IJCSI International Journal of Computer Science Issues, vol. 9, 2012.

[18] M. Kass, A. Witkin, and D. Terzopoulos, "Snakes: Active contour models," International Journal of Computer Vision, vol. 1, pp. 321-331, 1988.

[19] N. OTSU, "A Threshold Selection Method from Gray-Level Histograms," IEEE Transactions on Systems, Man and Cybernetics, vol. 9, pp. 62-66, 1979.

[20] C. Hollitt, "Reduction of computational complexity of Hough transforms using a convolution approach," in Proc. of 24th International Conference on Image and Vision Computing New Zealand, 2009, pp. 373-378.

[21] H. B. Qin, X. L. Wang, M. J. Liang, and W. H. Yan, "A novel pupil detection algorithm for infrared eye image," in Proc. of 2013 IEEE International Conference on Signal Processing, Communication and Computing (ICSPCC), 2013, pp. 1-5.

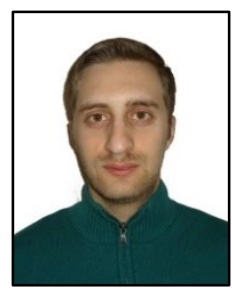

Mohammed A. M. Abdullah received the BSc and MSc degrees in computer engineering in 2008 and 2010 , respectively. Currently, he is studying toward the $\mathrm{PhD}$ degree in the School of Electrical and Electronic Engineering at Newcastle University, United Kingdom.

$\mathrm{He}$ worked as a research assistant in the Department of Electrical Engineering at the University of Liverpool, UK during 2010. His research interests are in the fields of pattern recognition and image processing.

M. Abdullah is a member of the IEEE and IET.

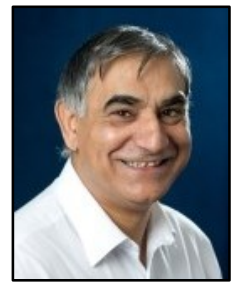

S. S. Dlay received the B.Sc. (Hons.) degree in Electrical and Electronic Engineering and the Ph.D. degree in VLSI design from Newcastle University, Newcastle upon Tyne, U.K., in 1979 and 1983, respectively.

During this time, he held a Scholarship from the Engineering and Physical Science Research Council (EPSRC) and the Charles Hertzmann Award. In 1984, he was appointed as a Post-Doctoral Research Associate at Newcastle University and helped to establish an Integrated Circuit Design Centre, funded by the EPSRC. In November 1984, he was appointed as a Lecturer in the Department of Electronic Systems Engineering at the University of Essex. In 1986, he re-joined Newcastle University as a Lecturer in the School of Electrical, Electronic, and Computer Engineering, then in 2001 he was promoted to Senior Lecturer. In recognition of his major achievements he has been appointed to a Personal Chair in Signal Processing Analysis. He has published over 250 research papers and his research interests lie in the mathematical advancement and application of modern signal processing theory to biometrics and security, biomedical signal processing and implementation of signal processing architectures. He serves on many editorial boards and has played an active role in numerous international conferences in terms of serving on technical and advisory committees as well as organizing special sessions.

Prof. Dlay is a College Member of the EPSRC.

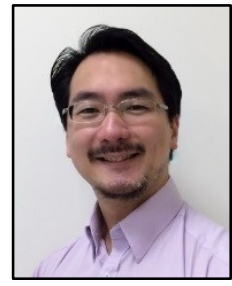

W. L. Woo received the BEng degree (1st Class Hons.) in Electrical and Electronics Engineering and the $\mathrm{PhD}$ degree from the Newcastle University, UK $\mathrm{He}$ is currently a Senior Lecturer with the School of Electrical and Electronics Engineering. His major research is in the mathematical theory and algorithms for nonlinear signal and image processing. This includes areas of machine learning for signal and image processing, blind source separation, and multidimensional signal processing.

He has published over 250 papers on these topics on various journals and international conference proceedings. Currently, he serves on the editorial board of several international journals and actively participate in international conferences and workshops, and serves on their organizing and technical committees.

Dr. Woo is a Senior Member of the IEEE. 